\title{
Utility of dobutamine stress echocardiography in aortic valve regurgitation and reduced left ventricular function
}

\author{
Chihiro Saito $^{1}$, Kotaro Arai ${ }^{1}$, Kyomi Ashihara $^{1}$, Hiroshi Niinami ${ }^{1}$, and Nobuhisa Hagiwara ${ }^{1}$ \\ ${ }^{1}$ Tokyo Women's Medical University
}

September 25, 2021

\begin{abstract}
Objective: Predictors for post-operative reverse remodeling in patients with severe aortic regurgitation (AR) and reduced left ventricular ejection fraction (LVEF) are unknown. We performed low-dose dobutamine stress echocardiography (DSE) in patients with severe AR and reduced LVEF to evaluate the relationship between contractile reserve (CR) and reverse remodeling after surgery. Methods: In 31 patients with chronic severe AR and reduced LVEF (LVEF < 50\%), we performed pre-operative DSE, assessed CR and examined whether changes in preoperative DSE were associated with improvement of post-operative LVEF after aortic valve surgery. Results: The pre-operative echocardiographic findings were as follows: left ventricular (LV) end-diastolic dimension: $67 \pm 10 \mathrm{~mm}, \mathrm{LV}$ end-systolic dimension: $52 \pm 13 \mathrm{~mm}$ and LVEF: $42 \% \pm 8 \%$. All patients underwent aortic valve surgery. Patients with pre-operative LVEF of $>45 \%$ exhibited a significant increase in LVEF; however, patients with pre-operative LVEF of $<45 \%$ showed no significant change. When we examined the results of DSE performed in patients with pre-operative LVEF of $<45 \%, \triangle \mathrm{LVEF}$ of [?] $6 \%$ (with CR) during DSE was related to an improvement in post-operative LVEF; $\triangle$ LVEF of [?] $6 \%$ during DSE predicted an improvement in post-operative LVEF, with a sensitivity and specificity of $80 \%$ and $85 \%$, respectively. Conclusions: DSE may be a helpful tool for predicting post-operative reverse remodeling in patients with severe AR and moderately reduced LVEF.
\end{abstract}

Original Research Article

Utility of dobutamine stress echocardiography in aortic valve regurgitation and reduced left ventricular function

Chihiro Saito ${ }^{1}$, MD, PhD; Kotaro Arai ${ }^{1}$, MD, PhD; Kyomi Ashihara ${ }^{1}, \mathrm{MD}$, PhD;

Hiroshi Niinami $^{2}$, MD, PhD; Nobuhisa Hagiwara ${ }^{1}, \mathrm{MD}, \mathrm{PhD}$

Department of Cardiology, Tokyo Women's Medical University, Tokyo, Japan.

Department of Cardiovascular Surgery, Tokyo Women's Medical University, Tokyo, Japan.

Address for correspondence: Kotaro Arai, $\mathrm{MD}, \mathrm{PhD}$

Department of Cardiology, Tokyo Women's Medical University,

8-1 Kawada-cho, Shinjuku-ku, Tokyo 162-8666, Japan

Tel: +81-3-3353-8111; fax: +81-3-3356-0441; e-mail: koh@wc4.so-net.ne.jp

\section{ABSTRACT}

Objective: Predictors for post-operative reverse remodeling in patients with severe aortic regurgitation (AR) and reduced left ventricular ejection fraction (LVEF) are unknown. We performed low-dose dobutamine 
stress echocardiography (DSE) in patients with severe AR and reduced LVEF to evaluate the relationship between contractile reserve $(\mathrm{CR})$ and reverse remodeling after surgery.

Methods: In 31 patients with chronic severe AR and reduced LVEF (LVEF < 50\%), we performed preoperative DSE, assessed CR and examined whether changes in preoperative DSE were associated with improvement of post-operative LVEF after aortic valve surgery.

Results: The pre-operative echocardiographic findings were as follows: left ventricular (LV) end-diastolic dimension: $67 \pm 10 \mathrm{~mm}$, LV end-systolic dimension: $52 \pm 13 \mathrm{~mm}$ and LVEF: $42 \% \pm 8 \%$. All patients underwent aortic valve surgery. Patients with pre-operative LVEF of $>45 \%$ exhibited a significant increase in LVEF; however, patients with pre-operative LVEF of $<45 \%$ showed no significant change. When we examined the results of DSE performed in patients with pre-operative LVEF of <45\%, $\triangle \mathrm{LVEF}$ of [?]6\% (with CR) during DSE was related to an improvement in post-operative LVEF; $\triangle$ LVEF of [?]6\% during DSE predicted an improvement in post-operative LVEF, with a sensitivity and specificity of $80 \%$ and $85 \%$, respectively.

Conclusions: DSE may be a helpful tool for predicting post-operative reverse remodeling in patients with severe AR and moderately reduced LVEF.

Key Words: aortic regurgitation, dobutamine stress echocardiography, aortic valve replacement, reverse remodeling

\section{Units and Abbreviations}

$\mathrm{AR}=$ aortic regurgitation

$\mathrm{CR}=$ contractile reserve

DSE $=$ dobutamine stress echocardiography

$\mathrm{EF}=$ ejection fraction

$\mathrm{LV}=$ left ventricular

$\mathrm{TTE}=$ transthoracic echocardiography

\section{Introduction}

Aortic regurgitation (AR) causes left ventricular (LV) volume and pressure overload. Initially, the left ventricle adapts to the volume overload with eccentric hypertrophy to preserve the LV ejection fraction (LVEF). ${ }^{12}$ However, progressive LV dilatation and pressure overload lead to myocardial damage and reduced ejection fraction (EF). Previous studies have reported that myocardial damage becomes irreversible even after aortic valve surgery, whereas some patients show significant improvement in LV function after surgery despite pre-operative LV dysfunction. ${ }^{3-5}$

The efficacy of aortic valve surgery in high-risk patients with severe AR and low LVEF is not well established. A recent study revealed that AVR was associated with lower mortality than medical therapy. ${ }^{6}$ This finding suggests that some of the pathophysiological changes in LVEF imposed by chronic severe AR are better tolerated than previously thought and that reverse remodeling can be achieved even in the late stages. Another study reported that post-operative and pre-operative reduced LVEF were associated with poor long-term outcomes. ${ }^{7}$ Therefore, there is a clinical need to predict LV reverse remodeling after aortic valve surgery.

Dobutamine stress echocardiography (DSE) is an established tool for detecting myocardial viability, ${ }^{8}$ and DSE has often been used to predict outcomes in various diseases. ${ }^{9-11}$ However, few reports have investigated the myocardial viability by DSE in patients with severe AR. Therefore, we performed low-dose DSE (LDSE) in patients with severe AR and reduced LVEF and examined the relationship with reverse remodeling after surgery. 


\section{Methods}

\section{Patients}

This retrospective observational study included 38 patients with chronic severe AR and reduced LVEF (LVEF $<50 \%$ ) for whom surgery had been indicated. We performed LDSE before surgery at Tokyo Women's Medical University Hospital, Japan, from July 2011 to November 2014. The severity of AR was diagnosed and graded by Doppler and colour Doppler echocardiography. ${ }^{11}$ Regarding indications for surgery, we followed the American Heart Association/American Colleague of Cardiology and European Society of Cardiology guidelines, in which surgery is recommended for all symptomatic patients and asymptomatic patients presenting severe AR and impaired function at rest (pre-operative LVEF < 50\%). ${ }^{12}{ }^{13}$ Patients with acute AR, coronary artery disease, aortic stenosis, significant mitral stenosis and regurgitation or previous aortic and mitral valve surgery were excluded. Of the 38 patients, 31 were included in our final analysis, excluding 3 who did not undergo surgery and 4 who had insufficient echocardiographic data after surgery.

The clinical parameters, including the New York Heart Association functional class, were recorded. Echocardiography, including LDSE, was performed in the pre-operative period, and follow-up echocardiography was performed in the late post-operative period [within 4.6 (range, 2.7-5.7] years].

For valve origin disease, aortic valve replacement with a bioprosthesis (15 patients) or a mechanical prosthesis (13 patients) was performed. For aortic root origin disease, Bentall operation (3 patients) was performed.

This study was conducted in accordance with the principles of the Helsinki Declaration. The review board of Tokyo Women's Medical University Hospital approved the protocol.

\section{Echocardiography}

A comprehensive transthoracic echocardiography (TTE), which included two-dimensional, M-mode, Doppler echocardiography was performed according to the recommendation of the American Society of Echocardiography. ${ }^{14}$ TTE examinations were performed using a Vivid 7 Ultrasound System (GE Healthcare, Horten, Norway). From the parasternal long axis view, we recorded the LV end-diastolic and end-systolic diameters. The LV end-diastolic volume (EDV), LV end-systolic volume (ESV) and LVEF were calculated using the biplane method of the disk summation. The stroke volume was calculated using the outflow tract diameter measured at the base of the aortic leaflet in the parasternal long axis view and the pulsed Doppler velocity integral obtained at the same level in the five-chamber or apical long axis view.

LDSE was performed before surgery. Dobutamine was infused intravenously in 10-min stages at incremental doses of 5 and $10 \mathrm{~g} / \mathrm{kg} / \mathrm{min}$ while monitoring a single-lead electrocardiogram and blood pressure. EDV, ESV, LVEF and stroke volume were obtained at each stage.

\section{Endpoints}

For the purpose of reverse remodeling after surgery, the primary endpoint of this study was improvement of the post-operative LVEF.

\section{Statistical analysis}

Results are presented as mean with standard deviation, median with interquartile range or frequency. The mean values were compared using paired $t$-test. Analysis of the receiver operating characteristic curve was used to assess the ability of $\triangle \mathrm{LVEF}$ during dobutamine infusion for detecting improvement of post-operative LVEF. The optimal cut-off point was calculated by determining the $\triangle$ LVEF during dobutamine infusion that provided the greatest sum of sensitivity and specificity. In all analyses, two-tailed $\mathrm{P}$ values of $<0.05$ were considered statistically significant. All analyses were performed with $\mathrm{R}$ statistical software ( $\mathrm{R}$ software, version 3.3.0; R Foundation for Statistical Computing, Vienna, Austria).

\section{Results}


The pre-operative demographic characteristics of the patients are shown in Table 1. A total of 31 patients were included (median age: 59 years; >90\% male). Nearly $80 \%$ patients had New York Heart Association functional class of $>$ II. Regarding the pre-operative echocardiographic parameters, the mean LV end-diastolic dimension (LVDd) was $67 \pm 10 \mathrm{~mm}$, the LV end-systolic dimension (LVDs) was $52 \pm 13 \mathrm{~mm}$, the LV enddiastolic volume index (LVEDVI) was $291 \pm 104 \mathrm{~mL} / \mathrm{m}^{2}$, the LV ESV index (LVESVI) was $98.5 \pm 40.2$ $\mathrm{mL} / \mathrm{m}^{2}$ and $\mathrm{LVEF}$ was $42 \% \pm 8 \%$. The aetiology of AR was degenerative disease in $25(80 \%)$ patients, bicuspid valve in $3(10 \%)$ and aortic dilatation in $3(10 \%)$.

All patients underwent the low-dose dobutamine protocol with no complications. No adverse effects were observed, and the $10 \mu \mathrm{g} / \mathrm{kg} / \mathrm{min}$ dose was administered in all patients. The comparison of echocardiographic variables at baseline (equally pre-operative) and during DSE with the $10 \mu \mathrm{g} / \mathrm{kg} / \mathrm{min}$ dose is shown in Table 2. Both LVEDVI and LVESVI during DSE decreased compared with those at baseline $(\mathrm{P}=0.015$ and $\mathrm{P}$ $<0.001$, respectively), and LVEF improved during DSE $(\mathrm{P}<0.001)$. The average $\triangle$ LVEF improvement was $8.9 \%$. The systolic blood pressure increased $(\mathrm{P}<0.001)$; however, the diastolic blood pressure did not change during DSE $(\mathrm{P}=0.787)$.

All patients underwent aortic valve surgery. The changes in LV volume (LVEDVI and LVESVI) and LVEF before and after surgery over a median follow-up period of $4.6(2.7-5.7)$ years are shown in Figure 1 . LV volume and function improved remarkably after surgery.

There were 18 patients with pre-operative LVEF of $>45 \%$ and 13 with pre-operative LVEF of $<45 \%$. Patients with pre-operative LVEF of $>45 \%$ exhibited a significant increase in LVEF (from $47.0 \pm 1.3 \%$ to $56.4 \pm 5.1$ $\%, \mathrm{P}<0.001$ ) (Figure 2). In contrast, in patients with pre-operative LVEF of $<45 \%$, there was no significant difference in LVEF (from $34.5 \pm 6.5 \%$ to $38.3 \pm 10.4 \%, \mathrm{P}=0.179$ ) (Figure 2).

Next, we examined the 13 patients with pre-operative LVEF of $<45 \%$ using the LDSE data. The receiver operating characteristic curve for improvement of post-operative LVEF indicated the cut-off point as $\triangle \mathrm{LVEF}$ of $6 \%$ during DSE. Of the 13 patients, 7 had $\triangle \mathrm{LVEF}$ of [?]6\% (with contractile reserve, CR) and 6 had $\triangle \mathrm{LVEF}$ of $<6 \%$ (without CR). The comparison of echocardiographic variables at baseline (equally pre-operative) and during DSE in patients with and without CR is shown in Table 3. In the patients with CR, there was no change in LVEDVI during DSE $(\mathrm{P}=0.54)$ and LVESVI during DSE decreased compared with that at baseline $(\mathrm{P}=0.002)$. In contrast, neither LVEDVI nor LVESVI changed in patients without $\mathrm{CR}(\mathrm{P}=0.09$ and $\mathrm{P}=0.13$, respectively).

All patients with CR $(\mathrm{n}=7$ ) had improvement in post-operative LVEF (from $33.0 \pm 7.0$ to $42.6 \pm 9.9 \%$, $\mathrm{P}=0.006$ ) (Figure 3). However, in six patients without CR, there was no change in LVEF (from $36.3 \pm$ 6.0 to $33.3 \pm 9.4 \%, \mathrm{P}=0.426$ ) (Figure 3). Patients with CR during DSE had a predicted improvement of post-operative LVEF with a sensitivity and specificity of $80 \%$ and $85 \%$, respectively.

\section{Discussion}

In this study, we performed DSE in patients with severe AR and reduced LVEF (pre-operative LVEF < $50 \%$ ), for whom surgery was indicated. We found that patients with pre-operative LVEF of $>45 \%$ exhibited a significant increase in LVEF; however, patients with pre-operative LVEF of $<45 \%$ showed no change in LVEF. When we examined the results of DSE performed in patients with pre-operative LVEF of $<45 \%$, $\triangle \mathrm{LVEF}$ of [?]6\% (with CR) during DSE was related to an improvement in post-operative LVEF.

Previous studies have shown that the long-term outcomes after AVR in patients with a low pre-operative LVEF are poor. ${ }^{15-17}$ Patients with reduced LVEF are often considered at very high risk of AVR, and some are denied surgery because they are thought to be at an irreversible worsening stage. However, a recent study revealed that a low pre-operative LVEF for AVR was associated with lower mortality than medical therapy. ${ }^{6}$ The post-operative echocardiographic parameters (LVDd, LVDs and LVEF) are important predictors of long-term cardiac death. ${ }^{7}$ In addition, some studies have shown that pre-operative LVEF does not correlate with the post-operative LVEF. Even when the pre-operative LVEF is depressed, the LVEF of some patients recovered after surgery and the long-term prognosis after AVR is not affected by pre-operative LV 
dysfunction. ${ }^{18} 19$ Therefore, it is clinically important to assess the viability and to identify the predictors of post-operative LV remodeling in patients with reduced pre-operative LVEF.

Some studies have focused on the effect of pre-operative status on recovery of LV function. Cho et al. demonstrated that an LVDs index of $<35.3 \mathrm{~mm} / \mathrm{m}^{2}$ and an LVDd index of $<44.2 \mathrm{~mm} / \mathrm{m}^{2}$ were associated with normalised LVEF (LVEF > 50\%) in 79 patients with severe AR who underwent AVR. However, in this study, 62 out of 79 patients had pre-operative LVEF of $>50 \% .{ }^{20}$ Furthermore, as for stress echocardiography for AR, CR by exercise echocardiography has been shown to be a prognostic value for post-operative LV function and clinical outcome in asymptomatic or mildly symptomatic patients with severe AR and preserved LVEF at rest. ${ }^{21}$ This exercise stress echocardiography also included patients with preserved LVEF. There are few studies regarding the predictions for post-operative LV remodeling that focus on patients with reduced pre-operative LVEF.

DSE is an established tool for detecting myocardial viability ${ }^{8}$ in various diseases, including chronic heart failure or aortic stenosis. ${ }^{9-11}$ However, there are few reports about DSE in patients with severe AR. Tam et al. studied 16 patients who underwent elective surgery for AR and had echocardiograms obtained at baseline and with $7.5 \mu \mathrm{g} / \mathrm{kg} / \mathrm{min}$ dobutamine. ${ }^{22}$ This study included patients with preserved or reduced LVEF; however, the authors concluded that LVEF during DSE was highly predictive of post-operative LVEF. DSE may therefore have a role in predicting the clinical outcomes of patients following AVR for AR. Marcia et al. performed DSE $(20 \mu \mathrm{g} / \mathrm{kg} / \mathrm{min})$ in 24 patients with AR and preserved LVEF (mean LVEF, $62.3 \%) .{ }^{23}$ However, they found that the percentage increase in LVEF under DSE did not predict the need for surgery and/or death. Unlike our study, this study included patients with preserved LVEF. Therefore, DSE may be better at identifying patients who already require surgery and are at a more advanced stage than asymptomatic or minimally symptomatic patients. These findings support our results that DSE may be a helpful tool for predicting post-operative reverse remodeling in patients with severe AR and, particularly, more progressive, decreased LVEF.

Our study is one of the few to investigate the usefulness of DSE in patients with severe AR and reduced LVEF and to show cut-off values for contractile reserve for the improvement of post-operative LVEF.

\section{Limitations}

The number of patients in this cohort was small, and the single-centre, retrospective, observational design of our study makes the establishment of causal relationships difficult. Further, our AR cohort was derived from a single tertiary referral centre in Japan and was thus subject to selection bias by including a highly selected population of patients with AR. We did not assess outcomes in this study. Therefore, a larger scale study with a larger number of patients and a longer period of follow-up and assessment of prognosis is needed to confirm our findings.

\section{Conclusions}

DSE may be a helpful tool for predicting post-operative reverse remodeling in patients with severe AR and moderately reduced LVEF.

Acknowledgments: We thank Edanz (https://jp.edanz.com/ac) for editing a draft of this manuscript.

Source of funding: None.

Conflict of interest disclosures: None.

\section{References}

1. Carabello BA. Aortic regurgitation. A lesion with similarities to both aortic stenosis and mitral regurgitation. Circulation 1990;82:1051-3.

2. Ferrari R, Ceconi C, Campo G, et al. Mechanisms of remodelling: a question of life (stem cell production) and death (myocyte apoptosis). Circ J 2009;73:1973-82. 
3. Bonow RO, Dodd JT, Maron BJ, et al. Long-term serial changes in left ventricular function and reversal of ventricular dilatation after valve replacement for chronic aortic regurgitation. Circulation 1988;78:1108-20.

4. Chaliki HP, Mohty D, Avierinos JF, et al. Outcomes after aortic valve replacement in patients with severe aortic regurgitation and markedly reduced left ventricular function. Circulation 2002;106:2687-93.

5. Enriquez-Sarano M, Tajik AJ. Clinical practice. Aortic regurgitation. N Eng J Med2004;351:1539-46.

6. Fiedler AG, Bhambhani V, Laikhter E, et al. Aortic valve replacement associated with survival in severe regurgitation and low ejection fraction. Heart 2018;104:835-40.

7. Amano M, Izumi C, Imamura S, et al. Pre- and Postoperative Predictors of Long-Term Prognosis After Aortic Valve Replacement for Severe Chronic Aortic Regurgitation. Circ J2016;80:2460-67.

8. Pierard LA, De Landsheere CM, Berthe C, et al. Identification of viable myocardium by echocardiography during dobutamine infusion in patients with myocardial infarction after thrombolytic therapy: comparison with positron emission tomography.J Am Coll Cardiol 1990;15:1021-31.

9. Parsai C, Baltabaeva A, Anderson L, et al. Low-dose dobutamine stress echo to quantify the degree of remodelling after cardiac resynchronization therapy. Eur Heart J2009;30:950-8.

10. Williams MJ, Odabashian J, Lauer MS, et al. Prognostic value of dobutamine echocardiography in patients with left ventricular dysfunction. J Am Coll Cardiol1996;27:132-9.

11. Zoghbi WA, Adams D, Bonow RO, et al. Recommendations for Noninvasive Evaluation of Native Valvular Regurgitation: A Report from the American Society of Echocardiography Developed in Collaboration with the Society for Cardiovascular Magnetic Resonance. J Am Soc Echocardiogr 2017;30:303-71.

12. Baumgartner H, Falk V, Bax JJ, et al. 2017 ESC/EACTS Guidelines for the management of valvular heart disease. Eur Heart $J$ 2017;38:2739-91.

13. Nishimura RA, Otto CM, Bonow RO, et al. 2014 AHA/ACC guideline for the management of patients with valvular heart disease: executive summary: a report of the American College of Cardiology/American Heart Association Task Force on Practice Guidelines. J Am Coll Cardiol 2014;63:2438-88.

14. Lang RM, Badano LP, Mor-Avi V, et al. Recommendations for cardiac chamber quantification by echocardiography in adults: an update from the American Society of Echocardiography and the European Association of Cardiovascular Imaging.J Am Soc Echocardiogr 2015;28:1-39 e14.

15. Forman R, Firth BG, Barnard MS. Prognostic significance of preoperative left ventricular ejection fraction and valve lesion in patients with aortic valve replacement. Am J Cardiol 1980;45:1120-5.

16. Henry WL, Bonow RO, Borer JS, et al. Observations on the optimum time for operative intervention for aortic regurgitation. I. Evaluation of the results of aortic valve replacement in symptomatic patients. Circulation 1980;61:471-83.

17. Kamath AR, Varadarajan P, Turk R, et al. Survival in patients with severe aortic regurgitation and severe left ventricular dysfunction is improved by aortic valve replacement: results from a cohort of 166 patients with an ejection fraction $<$ or $=35 \%$. Circulation 2009;120:S134-8.

18. Kang HJ, Kim YJ, Sohn DW, et al. Prediction of postoperative left ventricular systolic function with Doppler-derived dP/dt in patients with chronic aortic regurgitation.J Am Soc Echocardiogr 2003;16:1111-5.

19. Onishi T, Kawai H, Tatsumi K, et al. Preoperative systolic strain rate predicts postoperative left ventricular dysfunction in patients with chronic aortic regurgitation. Circ Cardiovasc Imaging 2010;3:134-41.

20. Cho SH, Byun CS, Kim KW, et al. Preoperative indexed left ventricular dimensions to predict early recovery of left ventricular function after aortic valve replacement for chronic aortic regurgitation. Circ $J$ 2010;74:2340-5. 
21. Park SJ, Enriquez-Sarano M, Song JE, et al. Contractile reserve determined on exercise echocardiography in patients with severe aortic regurgitation. Circ J2013;77:2390-8.

22. Tam JW, Antecol D, Kim HH, et al. Low dose dobutamine echocardiography in the assessment of contractile reserve to predict the outcome of valve replacement for chronic aortic regurgitation. Can J Cardiol 1999;15:73-9.

23. Barbosa MM, Freire CM, Fenelon LM, et al. Dobutamine-stress echocardiography in asymptomatic patients with aortic regurgitation. Arq Bras Cardiol 2009;93:53-8.

\section{Figure Legends}

FIGURE 1 Changes in echocardiographic data of 31 patients before and after surgery .

LVEDVI, left ventricular end-diastolic volume index; LVESVI, left ventricular end-systolic volume index; LVEF, left ventricular ejection fraction.

FIGURE 2 Changes in LVEF before and after surgery for patients with pre-operative LVEF of $>45 \%$ and patients with pre-operative LVEF of $<45 \%$.

In the box and whisker plots, the lines across each box represent the median LVEF; the box represents the interquartile range $\left(25^{\text {th }}-75^{\text {th }}\right.$ percentile) and the I bars represent the $95 \%$ confidence intervals.

LVEF, left ventricular ejection fraction.

FIGURE 3 Changes in LVEF before and after surgery in patients with and without contractile reserve $(\mathrm{CR})$.

In the box and whisker plots, the lines across each box represent the median LVEF; the box represents the interquartile range $\left(25^{\text {th }}-75^{\text {th }}\right.$ percentile) and the I bars represent the $95 \%$ confidence intervals.

CR, contractile reserve; LVEF, left ventricular ejection fraction.

Tables

Table 1 Baseline demographic characteristics

\begin{tabular}{ll}
\hline Variables & $\mathbf{N}=\mathbf{3 1}$ \\
\hline Men, n (\%) & $29(94)$ \\
Age, years & $59.4 \pm 14.5$ \\
Body surface area, ${ }^{3}$ & $1.74 \pm 0.15$ \\
NYHA functional class, n (\%) & \\
I & $7(23)$ \\
II & $15(48) ? \succsim ?$ \\
III & $9(29)$ \\
Systolic blood pressure, $\mathrm{mmHg}$ & $122.3 \pm 20.3$ \\
Diastolic blood pressure, $\mathrm{mmHg}$ & $53.9 \pm 14.6$ \\
Heart rate, bpm & $59.9 \pm 6.5$ \\
Echocardiogram & \\
LVDd, mm & $66.6 \pm 10.2$ \\
LVDd $/ \mathrm{BSA} . \mathrm{mm} / \mathrm{mm}^{2}$ & $38.3 \pm 4.7$ \\
LVDs, mm & $52.1 \pm 12.6$ \\
LVDs/BSA, mm $/ \mathrm{mm}^{2}$ & $29.9 \pm 6.0$ \\
LVEDV, $\mathrm{mL}$ & $290.8 \pm 103.6$ \\
LVEDVI, $\mathrm{mL} / \mathrm{m}^{2}$ & $165.8 \pm 52.5$ \\
LVESV, $\mathrm{mL}$ & $173.7 \pm 80.5$ \\
LVESVI, $\mathrm{mL} / \mathrm{m}^{2}$ & $98.5 \pm 40.2$
\end{tabular}




\begin{tabular}{ll}
\hline Variables & $\mathbf{N}=\mathbf{3 1}$ \\
\hline LVEF, \% & $41.8 \pm 7.6$ \\
Stroke volume, $\mathrm{mL}$ & $114.4 \pm 36.8$ \\
AR vena contracta, $\mathrm{mm}$ & $6.7 \pm 0.98$ \\
\hline
\end{tabular}

Values are mean \pm standard deviation or number (\%).

NYHA, New York Heart Association; BSA, body surface area; AR, aortic regurgitation; LVDd, left ventricular end-diastolic dimension; LVDs, left ventricular end-systolic dimension; LVEDV, left ventricular enddiastolic volume; LVEDVI, left ventricular end-diastolic volume index; LVEF, left ventricular ejection fraction; LVESV, left ventricular end-systolic volume; LVESVI, left ventricular end-systolic volume.

Table 2 Comparison of echocardiographic variables at baseline (equally pre-operative) and during dobutamine stress echocardiography

\begin{tabular}{|c|c|c|c|}
\hline Variables & Baseline & $\begin{array}{l}\text { During dobutamine } \\
\text { stress } \\
\text { echocardiography }\end{array}$ & $P$ value \\
\hline$\overline{\mathrm{LVEDV}}, \mathrm{mL}$ & $290.8 \pm 103.6$ & $273.3 \pm 96.0$ & 0.015 \\
\hline LVEDVI, $\mathrm{mL} / \mathrm{m}^{2}$ & $165.8 \pm 52.5$ & $155.6 \pm 47.7$ & 0.012 \\
\hline LVESV, mL & $173.7 \pm 80.5$ & $136.1 \pm 70.8$ & $<0.001$ \\
\hline LVESVI, $\mathrm{mL} / \mathrm{m}^{2}$ & $98.5 \pm 40.2$ & $77.0 \pm 35.2$ & $<0.001$ \\
\hline LVEF, \% & $41.8 \pm 7.6$ & $50.7 \pm 10.4$ & $<0.001$ \\
\hline Stroke volume, $\mathrm{mL}$ & $114.4 \pm 36.8$ & $116.7 \pm 36.4$ & $<0.001$ \\
\hline $\begin{array}{l}\text { Systolic blood pressure, } \\
\mathrm{mmHg}\end{array}$ & $122.3 \pm 20.3$ & $149.6 \pm 28.2$ & $<0.001$ \\
\hline $\begin{array}{l}\text { Diastolic blood } \\
\text { pressure, } \mathrm{mmHg}\end{array}$ & $53.9 \pm 14.6$ & $55.6 \pm 18.3$ & 0.787 \\
\hline Heart rate, bpm & $59.9 \pm 6.5$ & $74.0 \pm 13.1$ & $<0.001$ \\
\hline
\end{tabular}

Values are mean \pm standard deviation or number (\%).

LVEDV, left ventricular end-diastolic volume; LVEDVI, left ventricular end-diastolic volume index; LVEF, left ventricular ejection fraction; LVESV, left ventricular end-systolic volume; LVESVI, left ventricular endsystolic volume.

Table 3 Comparison of echocardiographic variables at baseline (equally pre-operative) and during dobutamine stress echocardiography

in patients with and without contractile reserve

\begin{tabular}{llll}
\hline Variables & Contractile reserve $(+)$ & Contractile reserve $(+)$ & Contractile reserve $(+)$ \\
\hline & Baseline & During DSE & P value \\
LVEDV, $\mathrm{mL}$ & $360.1 \pm 113.6$ & $353.7 \pm 118.1$ & 0.55 \\
LVEDVI, $\mathrm{mL} / \mathrm{m}^{2}$ & $196.9 \pm 51.0$ & $193.1 \pm 53.5$ & 0.54 \\
$\mathrm{LVESV}, \mathrm{mL}$ & $247.4 \pm 100.7$ & $213.4 \pm 89.0$ & 0.003 \\
$\mathrm{LVESVI}, \mathrm{mL} / \mathrm{m}^{2}$ & $134.6 \pm 47.1$ & $116.1 \pm 42.1$ & 0.002 \\
$\mathrm{LVEF}, \%$ & $33.0 \pm 7.0$ & $42.1 \pm 8.8$ & $<0.001$ \\
Stroke volume, $\mathrm{mL}$ & $100.0 \pm 29.6$ & $121.0 \pm 45.4$ & 0.07 \\
Systolic blood pressure, $\mathrm{mmHg}$ & $124.9 \pm 24.4$ & $155.6 \pm 27.9$ & 0.002
\end{tabular}




\begin{tabular}{llll}
\hline Variables & Contractile reserve $(+)$ & Contractile reserve $(+)$ & Contractile reserve $(+)$ \\
\hline Diastolic blood pressure, $\mathrm{mmHg}$ & $59.4 \pm 16.3$ & $61.1 \pm 15.1$ & 0.639 \\
Heart rate, bpm & $59.7 \pm 6.0$ & $78.3 \pm 8.3$ & 0.002 \\
\hline
\end{tabular}

Values are mean \pm standard deviation or number (\%).

CR, contractile reserve; DSE, dobutamine stress echocardiography; LVEDV, left ventricular end-diastolic volume; LVEDVI, left ventricular end-diastolic volume index; LVEF, left ventricular ejection fraction; LVESV, left ventricular end-systolic volume; LVESVI, left ventricular end-systolic volume.

\section{LVEDVI LVESVI LVEF}

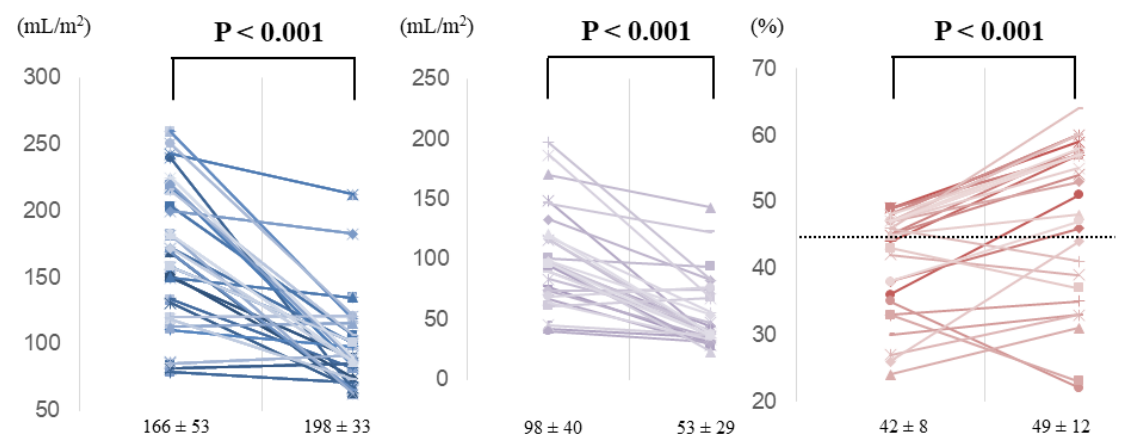

Pre-operative Post-operative Pre-operativePost-operative Pre-operative Post-operative

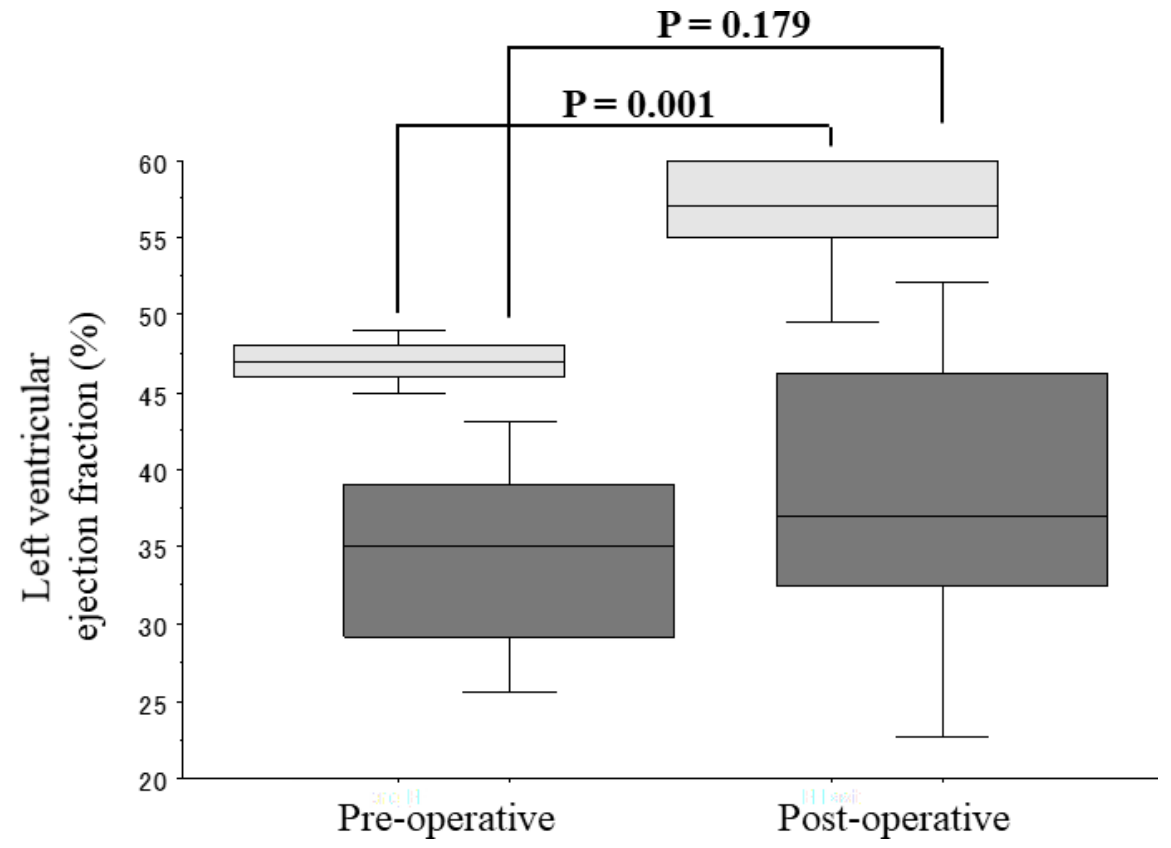


Patients with contractile reserve

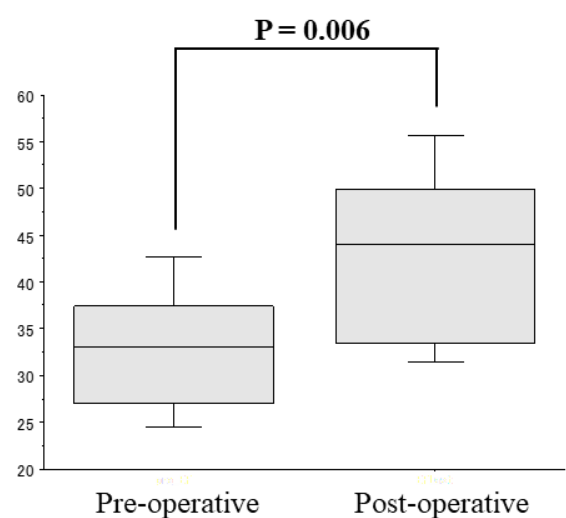

Patients without contractile reserve

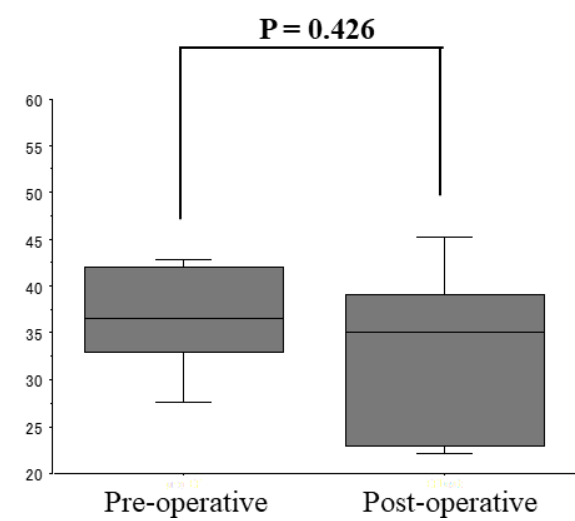

